\title{
Análisis comparativo del comportamiento de la escorrentía de tres microcuencas andinas con diferente régimen de precipitación y cobertura vegetal
}

\author{
Erika Martínez ${ }^{1}$, Cristian Coello ${ }^{1,2}$, Jan Feyen ${ }^{3}$ \\ ${ }^{1}$ Facultad de Ingeniería, Universidad de Cuenca, Av. 12 de Abril s/n, Cuenca, Ecuador. \\ 2 PROMAS, Programa para el Manejo del Agua y del Suelo, Universidad de Cuenca, Cuenca, \\ Ecuador. \\ ${ }^{3}$ Departamento de Recursos Hídricos y Ciencias Ambientales (iDRHICA), Universidad de Cuenca, \\ Cuenca, Ecuador.
}

Autor para correspondencia: erika.martinez2305@ucuenca.ec

Fecha de recepción: 3 de junio 2016 - Fecha de aceptación: 15 de enero 2017

\begin{abstract}
RESUMEN
Este artículo presenta un análisis descriptivo y comparativo de la precipitación y la escorrentía de tres microcuencas andinas (entre 0.6 y $1 \mathrm{~km}^{2}$ ) empleando aproximadamente 8 años de información, con el objetivo de relacionar las diferencias en el comportamiento del caudal con el nivel de precipitación anual, la distribución mensual de la lluvia y el uso del suelo. La cobertura de bosque nativo recibe en promedio la mayor cantidad de precipitación (1,540 mm/año), mientras que las otras dos microcuencas, cubiertas de pajonal con pastoreo extensivo y bosque de pinos respectivamente, reciben anualmente en promedio 1,267 y $1,182 \mathrm{~mm}$. A pesar de que el estudio no permitió describir a detalle los procesos que controlan la transformación de la precipitación en escorrentía, con el desglose exhaustivo de los datos de precipitación y escorrentía diarios sí se pudo definir el efecto del clima y la cobertura de suelo en los patrones de lluvia, para cada una de las microcuencas estudiadas. Además, este análisis a detalle permitió identificar con precisión los diferentes tipos de eventos, por lo que las microcuencas estudiadas muestran una respuesta diferente en la escorrentía relacionada con la diferencia en el clima y el uso del suelo, lo cual no hubiera sido posible de definir empleando únicamente los datos de precipitación promedio mensual y la información de escorrentía de las microcuencas, que es un enfoque tradicional que se emplea en los servicios de consultoría hidrológicos.
\end{abstract}

Palabras clave: Precipitación, escorrentía, cobertura de suelo, bosque nativo, bosque de pinos, páramo.

\begin{abstract}
The paper presents a descriptive comparative analysis of the precipitation and runoff of three Andean small watersheds (varying in size between 0.6 and $1 \mathrm{~km}^{2}$ ), using approximately 8 years of rainfall and discharge data, with the objective to relate differences in runoff behavior to the level of annual precipitation, the monthly distribution of rainfall and land use. The catchment covered with native forest receives on average the largest amount of rainfall $(1,540 \mathrm{~mm} / \mathrm{year})$, whereas the two other catchments, respectively under grassland with extensive cattle breeding and pine trees, receive annually on average respectively 1,267 and $1,181 \mathrm{~mm}$. Although the analysis did not permit to describe in detail the processes controlling the transformation of precipitation into runoff, the exhaustive breakdown of the daily rainfall and runoff data enabled to define the effect of climate and land cover on the runoff pattern of each of the studied micro-catchments. Furthermore, the detailed analysis allowed to accurately identify the type of events whereby the studied micro-catchments show a different response in runoff related to a difference in climate and land use, which would not have been possible to define using only the average monthly precipitation and runoff data of the catchments, a traditional approach in hydrologic consulting services.
\end{abstract}

Keywords: Precipitation, runoff, land cover, native forest, pine forest, páramo. 


\section{INTRODUCCIÓN}

El proceso lluvia-escorrentía y los modelos ideados para su estudio han sido usados para varios proyectos de planificación y gestión de recursos en todo el mundo, dada la necesidad de conocer la disponibilidad de los recursos hídricos y cómo varían estos en el tiempo con el fin de evaluar el impacto que el cambio climático o de uso del suelo podrían generar en la respuesta del caudal en los ecosistemas. A pesar de los esfuerzos que se han realizado los últimos años por conocer más a detalle los ecosistemas andinos, es todavía necesario enfatizar en el monitoreo y en el estudio de los mismos. Por esta razón, varios centros de investigación a nivel nacional e internacional continúan trabajando con el fin de aportar conocimiento que permita mejorar la eficiencia en el manejo y conservación de estos recursos tan importantes para la vida y los seres humanos. En el caso de PROMAS ${ }^{1}$, varias microcuencas son monitoreadas desde hace aproximadamente 8 años, contando en la actualidad con datos que permiten continuar y corroborar las investigaciones realizadas anteriormente.

La respuesta hidrológica de una cuenca está influenciada por el tipo y estado de la vegetación existente, las propiedades del suelo, su geomorfología, clima y uso del suelo; los cuales están influenciados principalmente por las actividades humanas (Knox, 2001; Jiménez, 2005) y el clima (Tomer \& Schilling, 2009). Varios estudios se han enfocado en cómo el clima y la cobertura de suelo interactúan (Copeland et al., 1996; Bonan, 1997; Pan et al., 1999; Mao \& Cherkauer, 2009), y en el impacto hidrológico de los cambios de uso de suelo (Mahe et al., 2004 citado en Sriwongsitanon \& Taesombat, 2011; Lin et al., 2007). El desarrollo de un enfoque integral, que permita simular y estimar los impactos de los cambios de uso de suelo y sus efectos en los procesos hidrológicos a nivel de cuenca, es crucial para la planificación y el manejo de los recursos hídricos, y para definir la influencia de la escorrentía superficial y el peligro de inundaciones (Twine et al., 2004; Ghaffari et al., 2010). Por ello, para un manejo efectivo de las cuencas, y su posterior restauración ecológica, es necesario conocer a detalle los procesos hidrológicos que en ellas ocurren. Además, es primordial conocer la influencia del cambio de uso de suelo en el balance hídrico, puesto que tal cambio afecta al tipo de cobertura del suelo, altera la escorrentía superficial y al resto de procesos hidrológicos de la cuenca (Brown et al., 2005).

Para analizar los efectos hidrológicos del impacto del cambio de uso de suelo y cambio climático, se han desarrollado principalmente tres métodos: estudio de cuencas pareadas, análisis de series de tiempo (método estadístico) y modelación hidrológica (Li et al., 2009). El enfoque de cuencas pareadas se considera como el mejor método para compensar la variabilidad climática en pequeñas cuencas experimentales; sin embargo, es difícil aplicar este método a cuencas medianas y grandes (Lørup et al., 1998). Así, las cuencas pequeñas $\left(<1,000 \mathrm{~m}^{2}\right)$ son una escala conveniente para la investigación, planificación y conservación del uso del suelo, cuantificar el potencial de los recursos hídricos y desarrollar estrategias apropiadas para el desarrollo de la agricultura en las llanuras de inundación, ya que se pueden identificar fácilmente en mapas y permiten descripciones detalladas de algunos procesos de los ecosistemas y sus capacidades (FAO, 2002; Fang et al., 2012; Wagner et al. , 2013, Han et al., 2012). El análisis de series de tiempo es un método estadístico fácil de aplicar, pero el estudio de los efectos hidrológicos es limitado debido a la falta de un mecanismo físico (Li et al., 2009). Con el fin de evaluar los impactos del cambio de uso de suelo en los recursos hídricos de manera cuantitativa y estudiar los impactos del cambio climático en la escorrentía y los sedimentos, se emplean normalmente modelos hidrológicos, los cuales son muy útiles puesto que permiten evaluar diferentes escenarios de uso de suelo, analizando así tanto impactos pasados como posibles impactos futuros. Los modelos más usados comúnmente son los modelos de regresión estadística, los modelos de balance hídrico y los modelos físicos distribuidos ( $\mathrm{Li} \&$ Gao, 2015). Para explorar los cambios de uso de suelo han sido explorados otros tipos de modelos tales como los modelos estocásticos, modelos de optimización, modelos dinámicos, modelos basados en simulación y modelos empíricos (p.e., Agarwal et al., 2002; Parker et al., 2002; Luijten, 2003; Rounsevell et al., 2003; Parker \& Meretsky, 2004; Wang et al., 2004; Stewart et al., 2004; citados en Lin et al., 2007). En estos modelos, las propiedades relevantes de la cobertura del suelo tienen que estar caracterizadas por parámetros relacionados al uso del suelo y al tipo de cultivo (Twine et al., 2004). Alrededor del mundo, también han sido llevados a cabo varios estudios regionales acerca de los efectos de los usos del suelo, empleados en el pasado, en la disponibilidad del

${ }^{1}$ PROMAS: Programa para el Manejo del Agua y del Suelo, Universidad de Cuenca. 
agua (Wagner et al., 2013). Según Li et al. (2009) el cambio de uso del suelo puede resultar en un cambio de frecuencia de inundaciones (Brath et al., 2006; Crooks \& Davies, 2001), severidad (De Roo et al., 2001), flujo base (Wang et al., 2006), y descarga media anual (Costa et al., 2003). Los efectos hidrológicos simulados para diferentes escenarios de uso de suelo son fundamentales para las decisiones que pretenden optimizar las funciones paisajísticas (Haverkamp et al., 2005). Li et al. (2009) también indica que con los métodos mencionados anteriormente se han realizado investigaciones en diferentes escalas de espacio y tiempo, en las cuales se ha concluido que el impacto de uso del suelo en los caudales pico ha sido generalmente más pronunciado a pequeñas escalas (Tollan, 2002). Por otra parte, el impacto del cambio de uso del suelo en el balance hídrico anual ha sido relativamente pequeño a grandes escalas de cuencas (Fohrer et al., 2001). Sin embargo, los impactos a corto plazo del cambio de uso del suelo y variación climática se han observado en los caudales pico, mientras que los impactos a largo plazo han sido más notables en la escorrentía promedio anual (Brath et al., 2006; Costa et al., 2003; Prowse et al., 2006). A pesar de que los efectos hidrológicos del cambio de uso de suelo y de la variación climática ocurren en todas las escalas espaciales, los estudios a escala local y regional son relevantes ya que proporcionan información importante a la economía local, al desarrollo social y a la protección ambiental (Lahmer et al., 2001).

Así, con el fin de contribuir al conocimiento acerca de la hidrología de los ecosistemas de alta montaña y complementar las investigaciones realizadas previamente en tres microcuencas andinas de alta montaña del sur del Ecuador (p.e., Célleri et al., 2004; Cisneros et al., 2007; Coello et al., 2008; Krajenbrink, 2007), los objetivos de este estudio son: examinar los cambios en la distribución de frecuencia de precipitaciones y caudales diarios durante un período de alrededor de 10 años, dividiendo la información en diferentes clases de acuerdo a la magnitud. Además, se llevará a cabo la realización del análisis hidrológico, separación de flujos y por último la definición de índices de conservación para la determinación del impacto del uso de suelo en las microcuencas.

\section{MATERIALES}

El área de estudio consiste en 3 microcuencas de alta montaña situadas en la zona central andina del Ecuador. La microcuenca Ortigas está cubierta en un $74 \%$ de bosque nativo, y está ubicada en la cuenca alta del río Bulu Bulu, en la provincia de Cañar. Se encuentra en las coordenadas $79^{\circ} 4^{\prime} \mathrm{O}$ y $2^{\circ} 25^{\prime} \mathrm{S}, \mathrm{y}$ cuenta con un área de $0.99 \mathrm{~km}^{2}$ (Fig. 1). La altitud en esta microcuenca varía entre 2,305 y 2,880 msnm, y tiene una pendiente media del $55 \%$. Presenta un régimen de lluvias occidental, y drena sus aguas hacia el océano Pacífico. La precipitación media anual en el período 2006-2011 varía entre 1,100 y 2,150 mm, produciéndose cerca del $90 \%$ de ésta entre los meses de diciembre y mayo. La temperatura media anual varía entre 13 y $15^{\circ} \mathrm{C}$. Las otras dos microcuencas son Pajonal y Pinos, llamadas así por su cobertura vegetal predominante, respectivamente de $86.4 \%$ de pajonal ${ }^{2}$ y $98 \%$ de Pinus patula. Ambas están ubicadas en el sector de El Cajas conocido como Marianza, en la provincia del Azuay. Pajonal se encuentra en las coordenadas $79^{\circ} 7^{\prime} \mathrm{O}$ Y $2^{\circ} 51^{\prime} \mathrm{S}$, y cuenta con un área de $1 \mathrm{~km}^{2}$; mientras que Pinos se encuentra en las coordenadas $79^{\circ} 6^{\prime} \mathrm{O}$ y $2^{\circ} 51^{\circ} \mathrm{S}$ y su correspondiente área es de $0.59 \mathrm{~km}^{2}$ (Fig. 1). Estas microcuencas drenan sus aguas hacia el Atlántico, y presentan un régimen de lluvias oriental. En Pajonal, la altitud varía entre 2,980 y 3,740 msnm con una pendiente media del $57 \%$, la precipitación media anual en el período 2008-2012 varía entre 1,100 y $1,550 \mathrm{~mm}$ y la temperatura media anual es de $9^{\circ} \mathrm{C}$. Por otro lado, la altitud en Pinos varía entre 3,240 y 3,700 msnm cuenta con una pendiente media del $50 \%$, su precipitación media anual en el mismo período varía entre 1,000 y 1,450 mm y la temperatura media anual está entre 8 y $10^{\circ} \mathrm{C}$.

\footnotetext{
2 Pajonal: Terreno cubierto de pajón y/o terreno bajo y anegadizo, cubierto de paja brava y otras especies asociadas, propias de los lugares húmedos.
} 

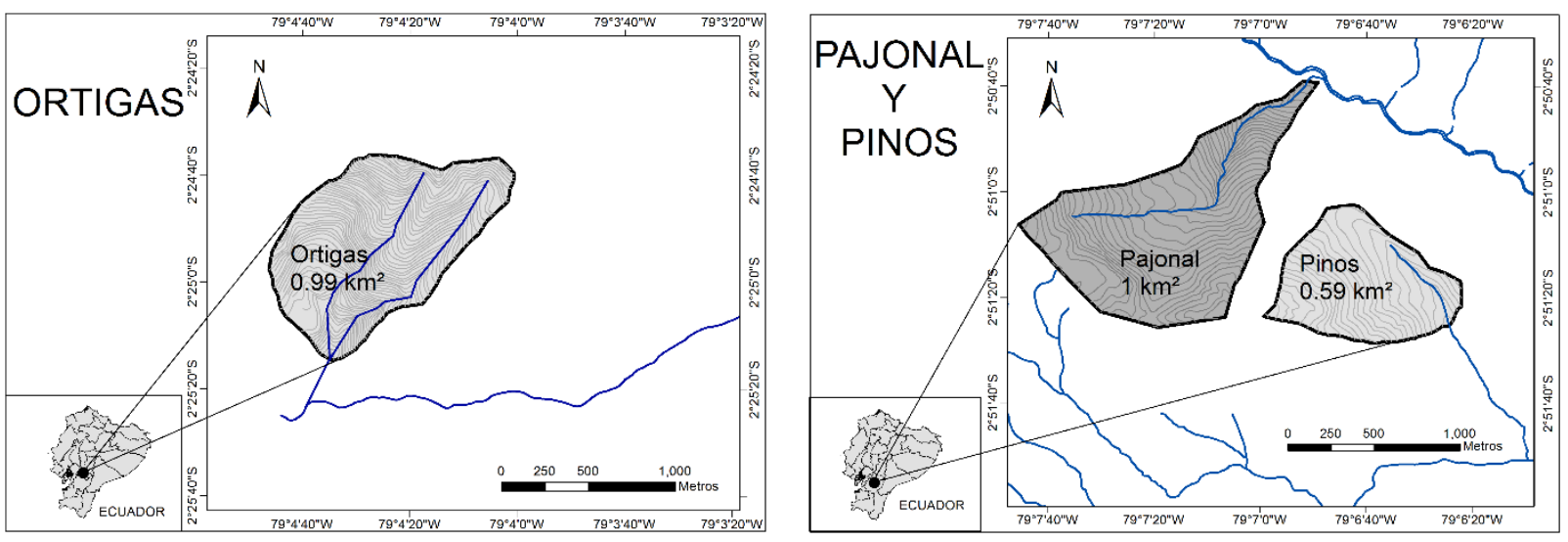

Figura 1. Ubicación de la microcuenca Ortigas (izquierda) y de las microcuencas Pajonal y Pinos (derecha).

El tipo de suelo predominante en las tres microcuencas es el Andosol úmbrico. Leptosoles también se han encontrado en Ortigas, aunque en áreas muy pequeñas. Los Andosoles son suelos desarrollados en materiales volcánicos que ocurren en todos los climas y todas las altitudes. Estos tipos de suelos poseen un alto potencial en la producción agrícola, ya que en general son suelos fértiles, tienen propiedades de almacenamiento de agua, baja capacidad portante y alta permeabilidad, lo que los hace resistentes a la erosión del agua (“Andosol," n.d.). La mayoría de Andosoles tienen un excelente drenaje interno debido a su alta porosidad y su predominancia en terrenos elevados, propiedades que explican su alta tasa de infiltración de agua bajo condiciones naturales (Harden, 1991; Nanzyo et al., 1993; Perrin et al., 2001). La mayoría de autores consideran que las singulares propiedades mineralógicas y el alto contenido de carbón orgánico de estos suelos son factores decisivos en sus propiedades estructurales (p.e., Caldas \& Salguero, 1975; Hoyos \& Comerford, 2005; Quantin, 1994; Warkentin \& Maeda, 1980 citado en Neris et al., 2013). Los Andosoles con horizonte úmbrico poseen un color de tierra desmenuzada y, en los primeros $20 \mathrm{~cm}$, una estructura suficientemente fuerte como para que el suelo no se vuelva masivo y duro cuando está seco ("El suelo es un ANDOSOL.," n.d.). Varios autores han prestado atención a la influencia ejercida en el comportamiento hidrológico de los Andosoles debido a características superficiales tales como cobertura vegetal (Cerdà, 1998, 1999; Molina et al., 2007), fragmentos rocosos (Descroix et al., 2001; Martínez-Zavala \& Jordán, 2008) e incluso ceniza (Cerdà \& Doerr, 2008; Woods \& Balfour, 2008, 2010; Zavala et al., 2009). Otros estudios acerca de los Andosoles también han destacado la degradación estructural y la reducción en la capacidad de infiltración producida por un cambio en el uso del suelo (p.e., Jiménez et al., 2006; Poulenard et al., 2001; Rodríguez et al., 2002; Warkentin \& Maeda, 1980; Zehetner \& Miller, 2006 citados en Neris et al., 2013). Otras características de las microcuencas de estudio se presentan en la Tabla 1.

Tabla 1. Características principales de las microcuencas.

\begin{tabular}{llll}
\hline & Ortigas & Pajonal & Pinos \\
\hline Uso del Suelo & Bosque nativo, & Ganadería Extensiva & Pinos \\
Estado & Inalterada & Poco alterada & Forestada \\
Forma de la Cuenca & Ovalada Alargada & Ovalada Alargada & Redonda-Ovalada \\
Perímetro (m) & 4,750 & 4,816 & 3,102 \\
Cota máxima (m s.n.m) & 2,880 & 3,740 & 3,700 \\
Cota mínima (m s.n.m) & 2,305 & 2,980 & 3,240 \\
Desnivel máximo (m) & 575 & 760 & 460 \\
Precipitación anual $(\mathrm{mm})$ & $1,540.6 \pm 348.3$ & $1,267.6 \pm 190.6$ & $1,181 \pm 182.5$ \\
Caudal anual (mm) & $8,17.5 \pm 341.7$ & $486.5 \pm 116.6$ & $319.8 \pm 98.2$ \\
Evapotranspiración $(\mathrm{mm})$ & $723.1 \pm 224.0$ & $781.1 \pm 110.8$ & $861.2 \pm 130.6$ \\
Coeficiente de escorrentía & $0.51 \pm 0.15$ & $0.37 \pm 0.37$ & $0.27 \pm 0.06$ \\
Caudal promedio diario $\left(1 / \mathrm{s} / \mathrm{km}^{2}\right)$ & $25.8 \pm 22.4$ & $16.0 \pm 12.0$ & $6.4 \pm 5.3$ \\
\hline
\end{tabular}




\section{MÉTODOS}

En primer lugar, se examinaron las características generales del clima en las tres microcuencas, tales como precipitación, caudal y evapotranspiración; además de la frecuencia de distribución de precipitaciones y caudales diarios durante 2005-2012 en Ortigas, y 2007-2013 en Pajonal y Pinos. En segundo lugar, con el fin de establecer una comparación entre los eventos de precipitación y caudal en los sitios de estudio, se realizó una clasificación del número de eventos y la cantidad de precipitación y caudal, siguiendo una metodología similar a la aplicada anteriormente por Han et al. (2012). En este caso se dividieron los datos en cinco grupos: eventos menores o iguales a $2 \mathrm{~mm} / \mathrm{día}$, entre 2 y $5 \mathrm{~mm} / \mathrm{día}$, entre 5 y $10 \mathrm{~mm} /$ día, entre 10 y $20 \mathrm{~mm} /$ día, y superiores a $20 \mathrm{~mm} /$ día. A continuación, se determinó el índice de Richard Baker en los años donde se disponía de la información requerida para el cálculo. Por último, se empleó la herramienta WETSPRO para la obtención de los sub flujos existentes en las microcuencas.

\subsection{Recolección y validación de datos}

Las tres microcuencas disponen de equipos de monitoreo hidrometeorológico implementados aproximadamente desde el año 2005 por PROMAS y gestionado por esta entidad hasta la actualidad. Los pluviógrafos son de cubeta basculante y tienen una resolución de $0.2 \mathrm{~mm}$; información que es procesada automáticamente a precipitaciones cada 5 minutos. Los vertederos de la salida de las microcuencas son triangulares y de cresta delgada. Mediante sensores de nivel ubicados en la sección de control se miden las alturas de flujo cada 15 minutos, las cuales son transformadas a caudal mediante ecuaciones conocidas de los vertederos triangulares. También están instaladas estaciones climatológicas para la medición de: velocidad y dirección del viento (anemómetro), radiación solar (piranómetro), y sensores para determinar temperatura, humedad relativa, presión barométrica y temperatura de rocío.

Los datos de precipitación, caudal y temperatura fueron empleados a escala diaria. Con el fin de no generar incertidumbre en los resultados se trabajó con información consistente de períodos de años consecutivos validados, resultando la serie de información de Ortigas de aproximadamente 7 años (01/08/2005-14/11/2012), y la serie de Pajonal y Pinos de aproximadamente 6 años (23/10/200710/09/2013). De esta manera se ignoraron vacíos considerables de información de caudal, debida a factores como la intervención de los habitantes de la zona, averías de los equipos de medición, mantenimiento de los sensores de nivel o limpieza de sedimentos acumulados. Las precipitaciones medias diarias fueron validadas mediante la aplicación de pruebas estadísticas no paramétricas como las curvas de doble masa, eliminando en cada caso datos dudosos que podrían deberse a errores de medición de los equipos. El relleno de precipitaciones faltantes fue mínimo, y para éste se emplearon análisis de regresión y técnicas de correlación.

\subsection{Indice de Richard Baker}

El índice de rapidez de respuesta de Richard Baker (índice R-B) definido por Baker et al. (2004), resulta una herramienta útil para determinar cambios graduales en el régimen de flujo asociados con cambios de uso y manejo del suelo, como los que se dan debido a la urbanización. En este caso se calculó con el objetivo de determinar la posible influencia de la vegetación sobre los caudales de las microcuencas. Este índice refleja la frecuencia y rapidez de los cambios a corto plazo producidos en el caudal, especialmente tras eventos de tormenta. Aumenta con el incremento de la frecuencia y magnitud de los eventos de lluvia y disminuye a medida que aumenta el flujo base y el área de la cuenca, pero presenta poca variabilidad interanual en relación con otros indicadores del mismo tipo (Baker et al., 2004). Varía año tras año debido a la variabilidad temporal de la precipitación, por lo que se debe disponer de aproximadamente 30 años de información para poder detectar tendencias en la rapidez de respuesta (OEHHA, 2015). Se calcula el índice R-B siguiendo la Ec. 1: 


$$
\text { Índice de rapidez de respuesta } R-B=\frac{\sum_{i=1}^{n}\left|q_{i}-q_{i-1}\right|}{\sum_{i=1}^{n} q_{i}}
$$

El método de cálculo mide la longitud de la trayectoria de las oscilaciones diarias de flujo para los datos de aforo del río. Trayectorias más largas se correlacionan con las corrientes más rápidas, mientras que flujos constantes tienen longitudes de trayectoria más cortas (Kotei et al., 2013). Los valores del índice R-B podrían variar teóricamente de cero a dos, y aumentan a medida que la longitud de la trayectoria y la velocidad aumentan (Baker et al., 2004). Si este valor es cercano a 0, significa que los caudales a lo largo del año hidrológico se mantuvieron estables, mientras que su incremento es símbolo de mayores fluctuaciones en los caudales a lo largo del año. Por lo tanto, tendría un valor de cero si el flujo de la corriente fuera absolutamente constante.

\subsection{Herramienta WETSPRO}

Se empleó la herramienta WETSPRO ${ }^{3}$ (Willems, 2004) para la realización de la separación de flujos en las tres microcuencas, con el fin de evaluar la influencia de la cobertura vegetal en la respuesta hidrológica del caudal a la precipitación en cada una. Este modelo calcula los flujos rápidos y lentos por medio de técnicas de separación de sub flujos basadas en la generalización del filtro original de Chapman; cuya técnica se basa en la ecuación general del "filtro de paso lento" y asume una recesión exponencial para los sub flujos (Willems, 2009 citado en Molina et al., 2012). Su interpretación física está basada en el concepto de modelo de reservorio lineal (ver Ec. 2; Willems, 2004).

$$
b(t)=\alpha b(t-1)+(1-\alpha)\left(\frac{q(t-1)+q(t)}{2}\right)
$$

donde: $\alpha=\exp \left(-\frac{1}{k}\right) ; q(t)=$ precipitación en la cuenca; $b(t)=$ escorrentía de la cuenca.

El parámetro $k$ es conocido como constante del reservorio o constante de recesión, y es un buen estimador de la capacidad de amortiguamiento de una cuenca (Célleri et al., 2004). Se puede determinar el valor de $k$ analizando la pendiente de los tramos de recesión en cada caso (flujo base, sub superficial, superficial) en los períodos de recesión, empleando la escala semi-logarítmica. Este valor controla qué tan lento el agua es desalojada del reservorio, y se define por características de la cuenca tales como la topografía, uso del suelo, tipo de suelo; las cuales no varían en el tiempo. Además, $k$ es menor para cuencas que están urbanizadas, tienen pendientes más altas o suelos más arenosos (Willems, 2004). Los parámetros $k \mathrm{y} w$ son los que se requiere determinar en el modelo WETSPRO:

- $1-w$ es el caudal que se convierte en flujo base; y

- $\quad k$ es el número de paso de tiempo que se demora el agua en ser desalojada de la cuenca.

Debido a que no todo el caudal se convertirá en caudal base, se debe emplear un factor de reducción $w$, que es un valor entre 0 y 1 e indica la fracción del caudal total que se convertirá en caudal base. Para determinar el valor de $w$ se debe considerar que el caudal base debe acercarse todo lo posible al caudal total durante las estaciones secas.

\section{RESULTADOS Y DISCUSIÓN}

Las microcuencas de los páramos ecuatorianos del sur tienen un clima húmedo perenne, típico de las regiones tropicales de alta montaña, con precipitaciones de baja intensidad extremadamente variables en espacio y tiempo (Buytaert, 2004). Debido a su localización cercana a la línea equinoccial, hay una

${ }^{3}$ WETSPRO: Water Engineering Time Series PROcessing software. 
baja variabilidad estacional de la temperatura media del aire, y la radiación solar diaria es prácticamente constante (Buytaert et al., 2006a), manteniéndose la temperatura en estas microcuencas alrededor de 8 y $10^{\circ} \mathrm{C}$ durante todo el año. Por otro lado, la microcuenca de Ortigas tiene una temperatura más elevada, entre 13 y $15^{\circ} \mathrm{C}$, debido a su menor altitud y su ubicación, más cercana a la costa del Pacífico. Dado que el lecho rocoso en las microcuencas es de tipo no fracturado, a diferencia de otros estudios como Han et al. (2012), es posible obtener los valores de evapotranspiración dados en la Tabla 1 a partir de la diferencia entre precipitación y caudal.

Varios autores sugieren que el impacto hidrológico de la cobertura de bosque varía fuertemente dependiendo del estado en el que se encuentra el ecosistema (Chazdon, 2008; Hofstede, 2011; Scott et al., 2005; Molina et al., 2012). Además, en Cantu \& Gonzalez (2005) se indica que la presencia de bosques en una cuenca hidrográfica no significa el aumento de la escorrentía en el caudal, sino, en muchos casos en función al tipo de bosque, su estado y el clima, su posible disminución como consecuencia de las demandas de agua por las plantas y su intercepción de la lluvia (evapotranspiración). Los valores de evapotranspiración fueron más altos en Pinos, seguidos por los de Pajonal y Ortigas. Esto se puede interpretar asumiendo que se dan mayores pérdidas por intercepción en la microcuenca de bosque de pinos, puesto que se trata de árboles relativamente jóvenes tal y como se ha demostrado en estudios como Farley et al. (2004), Hofstede (1998), Rutter (1959, 1963, 1967), y Corbett \& Crouse (1968).

Por otro lado, a pesar que la microcuenca de Ortigas es la que recibe mayor cantidad de precipitación, los valores de evapotranspiración son menores, debido a la presencia de bosque nativo maduro como tipo de vegetación predominante y menor necesidad de agua para la misma, como se ha demostrado en Bosch \& Hewlett (1982), Buytaert et al. (2007), y Farley et al. (2005), donde se indica que el rendimiento hídrico varía de acuerdo al tipo de vegetación y su estado de madurez, entre otras características. Dado que en Pajonal la evapotranspiración es menor que en Pinos, en esta microcuenca sus suelos también tendrán un menor grado de saturación. En Pajonal, tras cierto tiempo de invierno, el suelo empieza a saturarse, puesto que no es capaz de infiltrar más agua, mientras que en Pinos, debido a la mayor absorción de agua por parte de la vegetación, en el mismo período de invierno el suelo no está completamente saturado y el porcentaje de flujo superficial será menor.
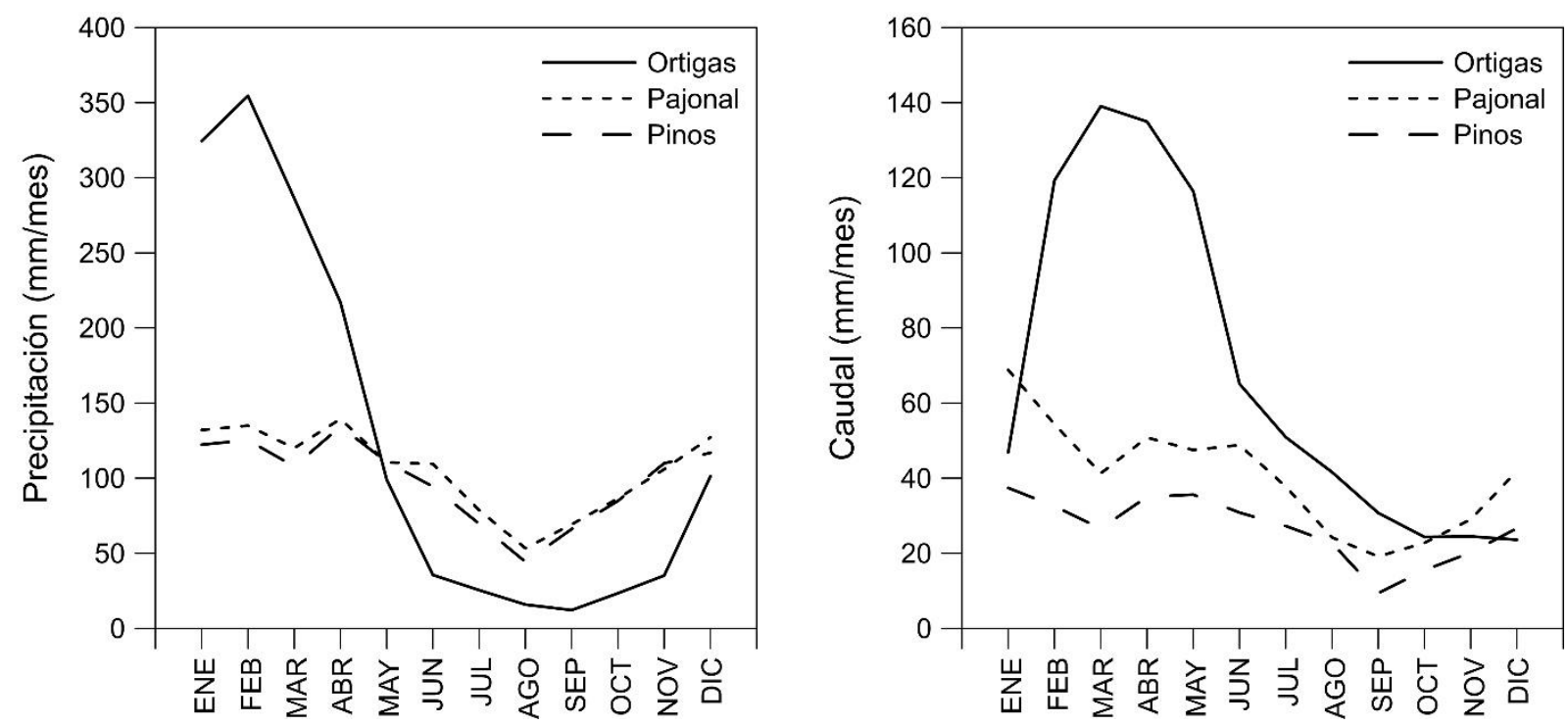

Figura 2. Promedio mensual de la precipitación (izquierda) y caudal (derecha) en mm, de las microcuencas Ortigas, Pajonal y Pinos.

Las distribuciones mensuales de las precipitaciones en las microcuencas de páramo mostrado en la Figura 2 son similares debido a su cercanía y siguen un régimen oriental, caracterizado por eventos constantes a lo largo del año. La cantidad de lluvia es mayor durante los meses de abril y enero, mientras que el menor valor de ésta se da en agosto. Por otro lado, en la microcuenca de bosque nativo el régimen 
de precipitaciones es occidental, definido por un fuerte invierno entre los meses de diciembre y mayo, siendo febrero el mes más lluvioso. En el período de junio hasta noviembre los eventos de precipitación son escasos.

La distribución mensual de caudales indica que la mayor cantidad del recurso se encuentra en Ortigas desde febrero hasta mayo, mientras que en las microcuencas de páramo los valores de caudal más altos se dan en los meses de enero, seguidos por los meses de abril, mayo y junio. Las microcuencas inalteradas poseen caudales característicos considerables, justificados por la escasa actividad humana existente en las mismas y la baja compactación del suelo. Además, según autores citados en Vuille (2013) y Andrade \& Ríos (2014), los ríos alimentados por páramos están caracterizados por la gran capacidad de retención de agua de los suelos y de la vegetación, además de un caudal base elevado. Las estaciones de invierno y verano en la microcuenca de Ortigas se distinguen en la Figura 2. Los caudales son más altos que los de las microcuencas de páramo, con valores mayores durante el invierno debido a la gran cantidad de precipitación existente, los cuales decrecen bruscamente en verano, a pesar que se mantienen elevados dada la buena capacidad de regulación que posee, a pesar de la gran cantidad de días sin lluvia. Por otro lado, en las microcuencas de páramo los caudales son más estables a lo largo del año y no hay variaciones tan fuertes, pero los valores son más bajos en la microcuenca de bosque de pinos debido a que este tipo de vegetación requiere de mayor cantidad del recurso, como se ha descrito en Célleri et al. (2004), Crespo et al. (2014) y Farley et al. (2005). Como referencia se determinó que el caudal específico mínimo en Pajonal es aproximadamente seis veces mayor al de Pinos.

La distribución de los eventos de precipitación es similar en los tres casos, a excepción de los eventos menores a $2 \mathrm{~mm} /$ día, donde se observó una diferencia significativa entre las microcuencas de páramo, en las que estos eventos son más frecuentes que en Ortigas, como se distingue en la Figura 3. Además, en el grupo de eventos mayores a $20 \mathrm{~mm} /$ día se refleja que los eventos de mayor magnitud se han producido en la microcuenca de bosque nativo, como los eventos extremos que se produjeron durante los inviernos de 2008 y 2009. También es posible observar, en la Figura 3, que la cantidad de lluvia de los eventos entre 10 y $20 \mathrm{~mm} /$ día es prácticamente la misma para las tres microcuencas, y que la diferencia es mayor nuevamente en los eventos mayores a $20 \mathrm{~mm} /$ día, debido también a los eventos extremos dados en Ortigas.

La forma de las curvas de duración general de caudales de la Figura 4 refleja la respuesta hidrológica específica que tienen las microcuencas de la presente investigación. La curva de duración de Ortigas es mucho más aplanada, similar a las curvas de duración de los ríos de llanura. Por debajo de esta se encuentra la curva de Pajonal, seguida por la de Pinos, indicando el aumento de intervención en las mismas. Las curvas de duración de Pajonal y Pinos tienen una forma similar, dada la regulación de caudales del páramo y la cobertura de bosque presente en la microcuenca de Pinos. La pendiente de la curva de duración de caudal de Pinos muestra un leve aumento con respecto a la de Pajonal, señal de la disminución en la capacidad de amortiguamiento del suelo (Buytaert et al., 2006b; Buytaert et al., 2007; Crespo et al., 2010). Sin embargo, esta alteración no cambia la forma del movimiento del agua dentro del ecosistema (Crespo et al., 2011 citado en Crespo et al., 2014). Por otro lado, Ortigas tiene una curva de duración con forma similar a las curvas de duración de cuencas de llanura, con una pendiente suave al inicio y caudales representativos, incluso durante las estaciones de verano (Cisneros et al., 2007).

Para poder establecer una tendencia de la variación de los caudales en las microcuencas se necesita como mínimo treinta años de información según OEHHA (2015). Al disponer únicamente de la tercera parte de esta información para definir el índice R-B, se pudieron calcular los valores iniciales de este índice que permiten tener una idea de la variación actual de los caudales en las microcuencas, mostrados en la Figura 5. En los tres casos estos valores resultaron ser relativamente bajos, demostrando que las condiciones en las tres microcuencas fueron bastante estables durante el período analizado, y por lo tanto las oscilaciones de los valores dados fueron debidas únicamente a las variaciones interanuales de la escorrentía. Li \& Gao (2015) indican que los cambios interanuales de la escorrentía pueden estar afectados también por factores tales como el cambio climático, actividades humanas y 
MASKANA, Vol. 8, No. 1, 2017
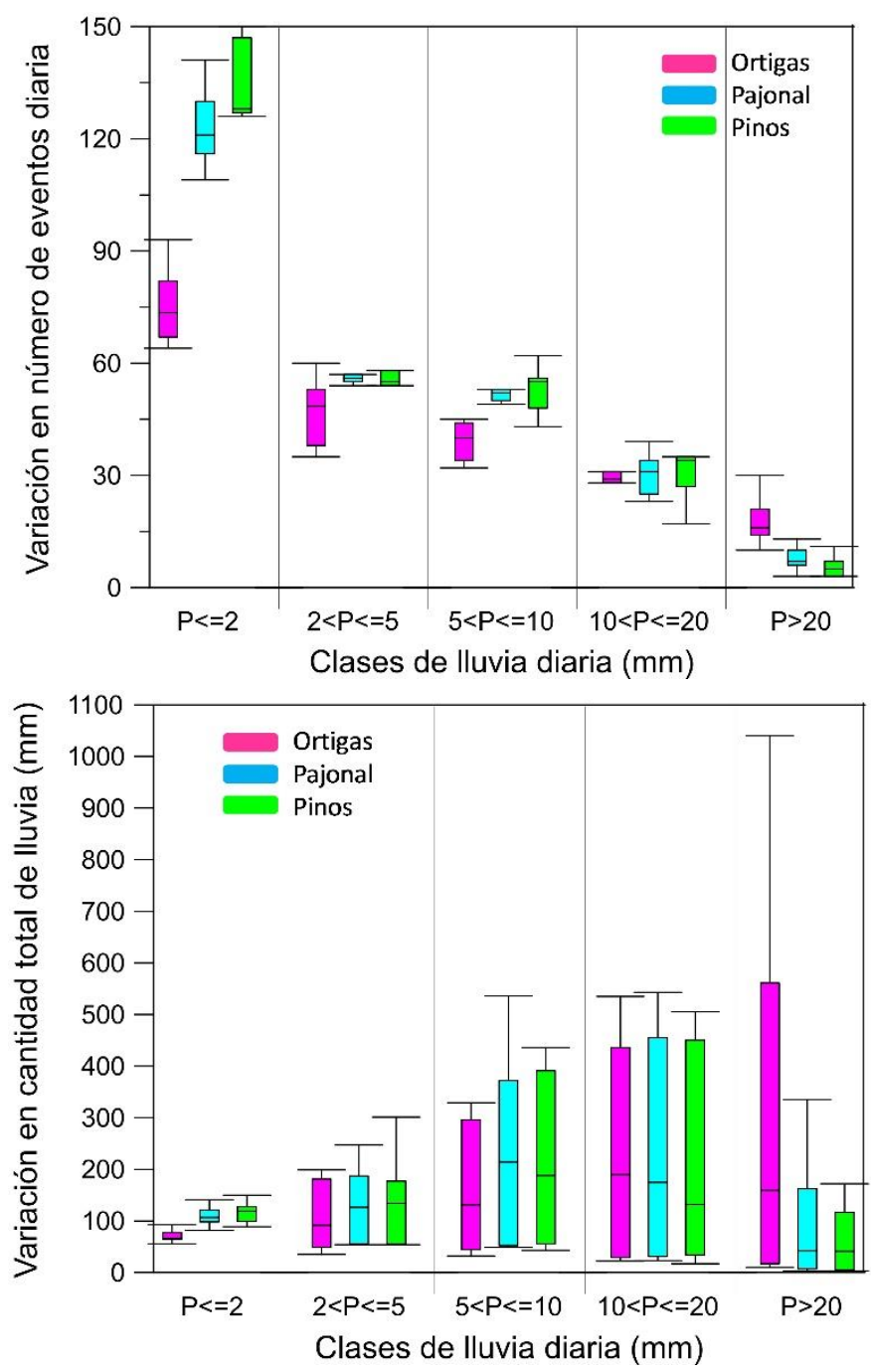

Figura 3. Distribución en número de eventos (arriba) y cantidad total de lluvia (mm) (abajo) por clase de lluvia diaria.

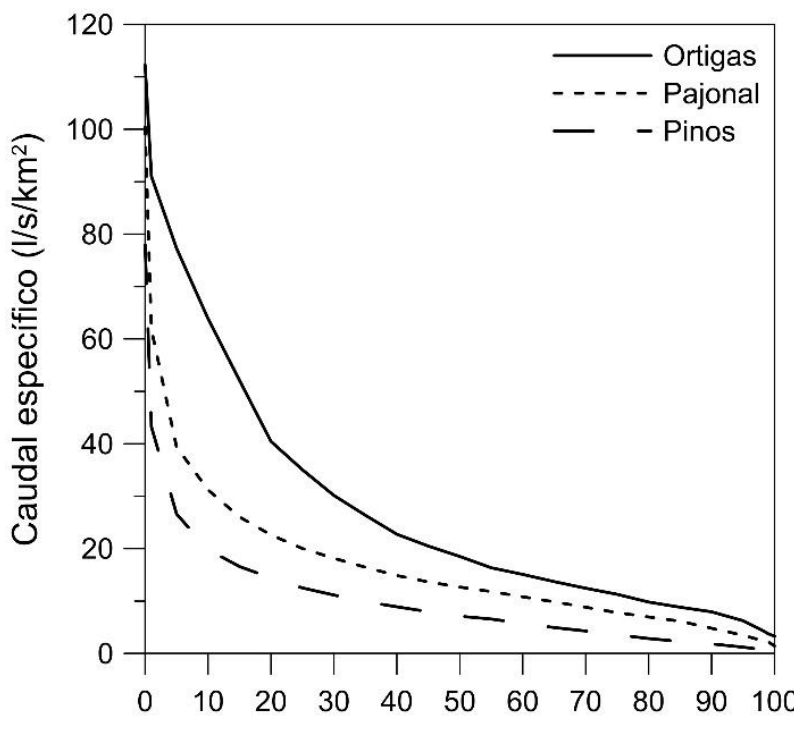

Probabilidad de excedencia (\%)

Figura 4. Curvas de duración de caudales. 
cambios en la superficie subyacente. Los valores más bajos del índice R-B se dieron en Ortigas y los más altos fueron los de Pajonal, en donde además de presentar mayores oscilaciones, estos valores son signo de un incremento de la densidad de vegetación en la microcuenca de Pajonal en los últimos años.

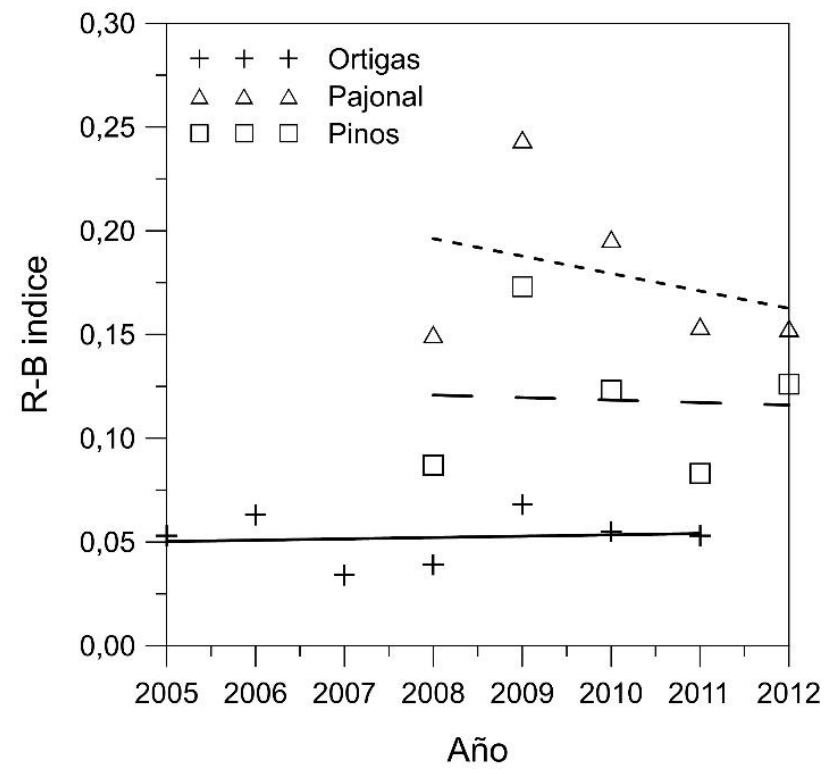

Figura 5. Variación anual del índice de rapidez R-B, respectivamente de las microcuencas Ortigas, Pajonal y Pinos.

Tabla 2. Resultados de la separación de flujos en las microcuencas de estudio.

\begin{tabular}{lccc}
\hline & Ortigas & Pajonal & Pinos \\
\hline \% Caudal base & $83.4 \pm 19.2$ & $67.7 \pm 19.7$ & $62.9 \pm 21.7$ \\
\% Caudal sub superficial & $9.5 \pm 10.8$ & $14.6 \pm 7.5$ & $22.4 \pm 12.9$ \\
\% Caudal superficial & $7.1 \pm 10.7$ & $17.7 \pm 18.7$ & $14.7 \pm 13.7$ \\
\hline
\end{tabular}

Tras la aplicación del modelo WESTPRO para la separación de flujos se establecieron los porcentajes de caudal base, sub superficial y superficial de cada microcuenca mostrados en la Tabla 2. Con los diagramas de caja obtenidos a partir de los porcentajes dados por el modelo, mostrados en la Figura 6, se observa que Ortigas es la microcuenca donde los valores de flujo base son más elevados, con un valor medio cercano al $90 \%$. Este valor es seguido por la microcuenca de Pajonal, donde el porcentaje medio del flujo base es del $70 \%$. Por último, Pinos tiene un valor de flujo base alrededor del $65 \%$. Es probable que la cantidad de flujo base que circula por la microcuenca de Pinos sea inferior al de las otras dos microcuencas debido a que en ella se produce una mayor intercepción a causa de su cobertura de pinos, la cual retiene mayor cantidad del recurso que las otras dos coberturas vegetales, puesto que la cantidad de escorrentía puede ser modificada debido a la cobertura de bosque como indican Guevara-Escobar et al. (2007). La mayor cantidad de flujo base en Ortigas es signo del efecto protector del suelo de bosque contra los procesos erosivos y la alta resistencia de los Andosoles a la erosión, bajo condiciones de bosque como indican Neris et al. (2013). Los valores de flujo superficial y sub superficial en las tres microcuencas son pequeños en comparación con los del flujo base. Pinos tiene el rango de caudales sub superficiales más elevados, con un valor medio alrededor del $22 \%$, seguido del $14 \%$ en Pajonal y $6 \%$ en Ortigas. Los porcentajes de caudal superficial en Pajonal y Pinos son similares, alrededor del 12\%, a pesar que en Pajonal se da una mayor dispersión de los mismos. En Ortigas el valor medio del flujo superficial es mucho más pequeño, alrededor del $2 \%$.

Debido a que WETSPRO (Willems, 2004) ha sido validado principalmente en ríos medianos a grandes, en áreas de pendiente plana a moderada, donde los tres componentes del flujo (base, subsuperficial y superficial) son claramente detectables, en la región andina del Ecuador el software debe ser usado con precaución ya que no es siempre fácil distinguir entre el flujo superficial y 
subsuperficial debido al horizonte orgánico rico y poroso de los Andosoles. Por lo tanto, como indica Crespo et al. (2010) es más correcto considerar, lo que WETSPRO genera como flujo superficial y subsuperficial, como un solo componente, o lo que es lo mismo, que los componentes de flujo superficial y sub superficial circulan a través de los horizontes orgánicos y el flujo base circula a través del horizonte mineral.

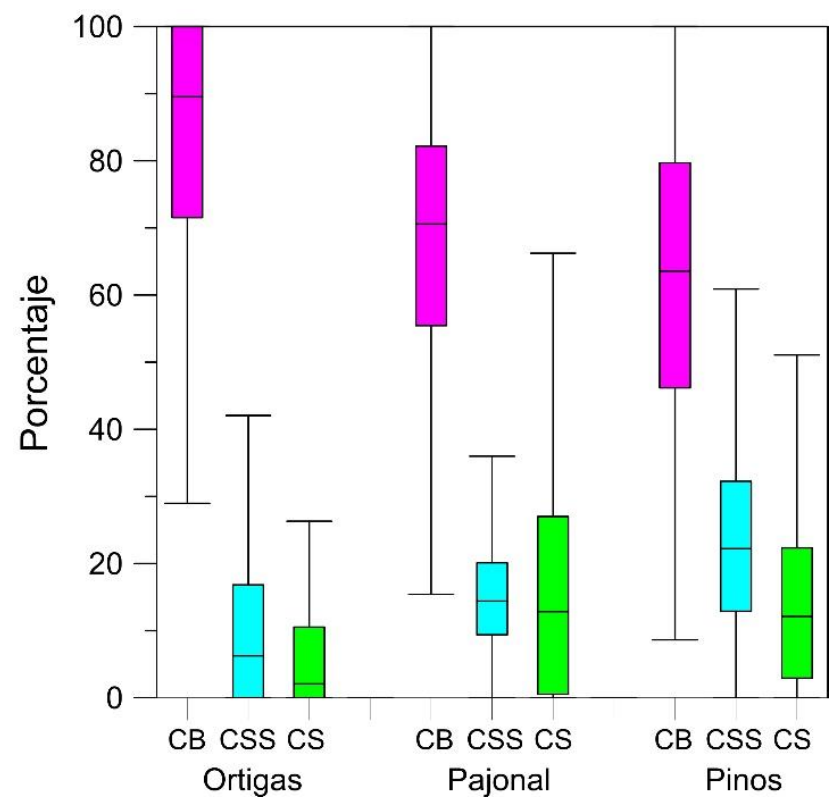

Figura 6. Diagrama de caja y bigotes de la fracción de caudal base (CB), caudal sub superficial (CSS) y caudal superficial (CS) de las microcuencas Ortigas, Pajonal y Pinos.

Así, debido al efecto que tiene la vegetación en estas microcuencas, se puede decir que los porcentajes de flujo superficial dados por el modelo en realidad no representan el flujo que circula sobre el suelo, sino que es más probable que este flujo superficial circule a través del horizonte orgánico, y que se trate de una especie de flujo sub superficial. Al tratarse de Andosoles como tipo de suelo predominante, caracterizados por sus horizontes orgánicos y alta porosidad, los cuales brindan a las microcuencas condiciones favorables y mayor capacidad de infiltración, es más probable que la suma de los porcentajes de flujo superficial y sub superficial sea la fracción total de flujo que circule a través del horizonte orgánico. Además, puesto que se trata de vegetaciones tales como bosque nativo, bosque de pinos y pajonales es posible pensar que se haya dado una acumulación de materia orgánica en las capas superiores del suelo, lo que justifica la alta porosidad de la parte alta del horizonte mineral. Por otro lado, el porcentaje de flujo base dado por el modelo sería aquel que circula a través del horizonte mineral. Los Andosoles tienen una porosidad muy alta, y cuando se dan precipitaciones fuertes, la mayoría del agua circula directamente hacia el sub suelo, lo que justificaría los altos porcentajes de caudal base obtenidos en los tres casos. Así, únicamente existiría flujo superficial en épocas de invierno, cuando el suelo se satura, y el agua realmente permanece en la superficie.

\section{CONCLUSIONES}

Tras analizar los resultados se pudo observar que la vegetación no causó mayor impacto en la hidrología de estas microcuencas, puesto que no se encontraron mayores diferencias en el comportamiento de las mismas. Esto es debido a que las tres microcuencas están prácticamente cubiertas en su totalidad por una vegetación característica desde hace bastante tiempo, incluso la microcuenca de Pinos, por lo que el impacto de la vegetación es bastante similar en las tres microcuencas. El efecto más notable en la hidrología de las microcuencas debido a la vegetación fue la diferencia en la evapotranspiración, lo cual afecta directamente a la escorrentía como en el caso de Pinos, donde los caudales fueron más bajos 
debido a este factor. Por otro lado, se puede afirmar que, desde el punto de vista del estado de conservación, las condiciones hidrológicas de estas microcuencas son estables a lo largo del período de estudio, lo cual se entiende debido a que la vegetación no varió notablemente a lo largo del tiempo, e incluso pueden ser usadas como modelo a seguir para la conservación de otras cuencas de montaña.

La microcuenca de Ortigas presenta caudales mínimos importantes a pesar de los períodos de sequía largos, como el de 150 días con precipitaciones diarias esporádicas menores a $2 \mathrm{~mm}$; y su rendimiento hidrológico específico es el más alto comparado con las otras microcuencas. Con la información disponible se pudo realizar el análisis de precipitación y caudal únicamente de manera descriptiva, lo que permitió detectar ciertas diferencias en el proceso lluvia-escorrentía de las microcuencas. El enfoque del análisis a detalle de la precipitación y la escorrentía permitió identificar con claridad la clase de eventos en los que se encuentran las diferencias de precipitación y caudal, lo cual no hubiera sido posible definir basándose únicamente en los gráficos de la Figura 2, que son con los que se trabaja y se obtiene conclusiones normalmente en el ámbito de la ingeniería. Con la información de la Figura 3 se pueden clasificar tres eventos distintos de precipitación: eventos de precipitación menores a $2 \mathrm{~mm} / \mathrm{día}$, que predominan en las microcuencas de páramo y son menos usuales en Ortigas; eventos entre 2 y 20 $\mathrm{mm} /$ día, que ocurren aproximadamente en cantidades iguales en las tres microcuencas; y eventos mayores a $20 \mathrm{~mm} / \mathrm{día}$, los cuales se han producido en mayor cantidad en Ortigas. Esta diferencia es consecuencia de la variación en el tipo y régimen de precipitaciones en Ortigas, a diferencia de las otras dos microcuencas de páramo.

Los resultados de la separación de flujos muestran que el caudal base es el mayor componente, sobre todo en Ortigas, donde se demuestra el efecto protector de la cobertura orgánica de bosque y cómo los Andosoles ayudan a contribuir a los caudales base debido a su alta porosidad. Esta microcuenca presenta condiciones hidrológicas óptimas, una buena capacidad de regulación de caudales, respuesta lenta ante eventos de precipitación y buena disponibilidad del recurso durante todo el año, a pesar de contar con seis meses de verano.

En Pinos existe un mayor flujo superficial y sub superficial; esta escorrentía determinada por el modelo WETSPRO es mayor que la de su cuenca vecina de cobertura vegetal propia de la zona. La combinación de la precipitación y de la vegetación juntamente con el flujo base hacen que, en efecto, exista una mayor capacidad de intercepción en la microcuenca de bosque de pinos, que implica una mayor pérdida del recurso debido al consumo de agua que presenta este tipo de vegetación exótica para la zona de páramo de El Cajas, como se ha demostrado en estudios previos (Buytaert et al., 2007; Célleri et al., 2004; Cisneros et al., 2007; Coello et al., 2008; Crespo et al., 2010). De igual manera, se ha mostrado que la cobertura vegetal no presenta ninguna influencia en la respuesta a los eventos extremos de períodos de retorno altos.

Los presentes resultados podrían ser complementados en futuras investigaciones. Para esto, es necesario recolectar más información base que permita usar modelos para realizar un análisis de escenarios de futuros usos del suelo y sus impactos en los recursos hídricos y de la influencia del cambio

climático en estas microcuencas. El conocimiento de estos aspectos no es trivial ya que la hidrología de las cuencas de alta montaña afecta directamente a la población y a los ecosistemas aguas abajo, al riego en la agricultura, a las industrias, entre otros.

\section{BIBLIOGRAFÍA}

Andosol (n.d.). Recuperado de http://www.isric.org/sites/default/files/ major_soils_of_the_world/ set3/an/andosol.pdf

Andrade L, Ríos C (2014). Variabilidad climática y caudales mínimos en los Andes Ecuatorianos. Revista Politécnica 33(1), 9 pp

Baker D, Richards R, Timothy T, Kramer J (2004). A new flashiness index: characteristics and applications to Midwestern rivers and streams. Journal of the American Water Resources Association 40(2):503-522

Bonan GB (1997). Effects of land use on the climate of the United States. Climate Change 37:449-486 
Bosch JM, Hewlett JD (1982). A review of catchment experiments to determine the effect of vegetation changes on water yield and evapotranspiration. Journal of Hydrology 55(1):3-23

Brath A, Montanari A, Moretti G (2006). Assessing the effect on flood frequency of land use change via hydrological simulation (with uncertainty). Journal of Hydrology 324(1-4):141-153

Brown DG, Johnson KM, Loveland TR, Theobald DM (2005). Rural land-use trends in the conterminous United States, 1950-2000. Ecological Applications 15(6)1851-1863

Buytaert W, De Bièvre B, Wyseure G, Deckers J, 2004. The use of the linear reservoir concept to quantify the impact of land use changes on the hydrology of catchments in the Ecuadorian Andes. Hydrology and Earth System Sciences 8:108-114

Buytaert W, Célleri R, De Bièvre B, Cisneros F (2006a). Hidrología del páramo andino: Propiedades, importancia y vulnerabilidad. Cuenca. Recuperado de http://www. paramo. org/files/hidrologia_paramo. pdf, $26 \mathrm{pp}$

Buytaert W, Célleri R, De Bièvre B, Cisneros F, Wyseure G, Deckers J, Hofstede R (2006b). Human impact on the hydrology of the Andean páramos. Earth Science Review 79(1):53-72

Buytaert W, Iniguez V, De Bievre B (2007). The effects of afforestation and cultivation on water yield in the Andean páramo. Forest Ecolpgy Management 251(1):22-30

Cantu I, Gonzalez H (2005). Pérdidas por intercepción de la lluvia en tres especies de matorral submontano. Ciencia de la Universidad Autónoma de Nuevo León 8(1), 6 pp

Célleri R, De Biévre B, Iñiguez V (2004). Efectos de la cobertura vegetal en la regulación hidrológica de microcuencas de páramo. Informe Final. Dirección de Investigación de la Universidad de Cuenca, $23 \mathrm{pp}$

Cerdà A (1998). Postfire dynamics of erosional processes under mediterranean climatic conditions. Zeitschrift für Geomorphologie 42(3):373-398

Cerdà A (1999). Parent material and vegetation affect soil erosion in eastern Spain. Soil Science Society of America Journal 63:362-368

Cerdà A, Doerr SH (2008). The effect of ash and needle cover on surface runoff and erosion in the immediate post-fire period. Catena 74:256-263

Chazdon RL (2008). Beyond deforestation: restoring forests and ecosystem services on degraded lands. Science 320(5882):1458-1460

Cisneros F, Coello C, Crespo P, De Bièvre B, Feyen J (2007). Análisis de curvas de duración general para determinar el grado de degradación de cuencas hidrográficas. Revista Anales de la Universidad de Cuenca, pp. 71-76

Coello C, Cisneros F, Feyen J (2008). Efecto de la Cobertura Vegetal en la Respuesta Hidrológica de Cuencas Hidrográficas. Revista Anales de la Universidad de Cuenca, pp. 65-70

Copeland JH, Pielke RA, Kittel TGF (1996). Potential climatic impacts of vegetation change: A regional modeling study. Journal of Geophysical Research 101:7409-7418

Corbett ES, Crouse RP (1968). Rainfall interception by annual grass and chaparral losses compared. Berkeley, Calif., Pacific SW. Forest \& Range Exp. Sta., U.S. Forest Serv. Res. Paper PSW-48, $12 \mathrm{pp}$

Costa MH, Botta A, Cardille JA (2003). Effects of large-scale changes in land cover on the discharge of the Tocantins River, Southeastern Amazonia. Journal of Hydrology 283:206-217

Crespo P, Célleri R, Buytaert W, Feyen J, Iñiguez V, Borja P, De Bièvre B (2010). Land use change impacts on the hydrology of wet Andean páramo ecosystems. Status and Perspectives of Hydrology in Small Basins. IAHS Publ 336:71-76

Crespo P, Célleri R, Buytaert W, Ochoa B, Cárdenas I, Iñiguez V, Borja P, De Bièvre B (2014). Impactos del cambio de uso de la tierra sobre la hidrología de los páramos húmedos andinos. En: Cuesta F, Sevink J, Llambí LD, De Bièvre B, Posner (Eds.), Avances en investigación para la conservación de los páramos andinos. CONDESAN, pp. 287-304 
Crooks SM, Davies HN (2001). Assessment of land use change in the Thames catchment and its effect on the flood regime of the river. Physics and Chemistry of the Earth, Part B: Hydrology, Oceans and Atmosphere 26(7-8):583-591

De Roo A, Odijk M, Schmuck C, Koster E, Lucieer A (2001). Assessing the effects of land use changes on floods in the Meuse and Oder catchments. Physics and Chemistry of the Earth, Part B: Hydrology, Oceans and Atmosphere 26(7-8):593-599

Descroix L, Viramontes D, Vauclin M, Gonzalez Barrios JL, Esteves M (2001). Influence of soil Surface features and vegetation on runoff and erosion in the Western Sierra Madre (Durango, Northwest Mexico). Catena 43(2):115-135

Fang N-F, Shi Z-H, Li L, Guo Z-L, Liu Q-J, Ai L (2012). The effects of rainfall regimes and land use changes on runoff and soil loss in a small mountainous watershed. Catena 99:1-8

FAO (2002). Land-water linkages in rural watersheds. Case Study Series, Food and Agriculture Organization of the United Nations, Rome, Italy. Disponible en ftp://ftp.fao.org/agl/aglw/docs/lw9e.pdf, 90 pp

Farley K, Jobbágy E, Jackson R (2005). Effects of afforestation on water yield: A global synthesis with implications for policy. Global Change Biology 11(10): 1565-1576

Farley K, Kelly E, Hofstede R (2004). Soil organic carbon and water retention after conversion of grasslands to pine plantations in the Ecuadorian Andes. Ecosystems 7(7):729-739

Fohrer N, Haverkamp S, Eckhardt K, Frede H (2001). Hydrologic response to land use changes on the catchment scale. Physics and Chemistry of the Earth, Part B: Hydrology, Oceans and Atmosphere 26(7-8):577-582

Ghaffari G, Keesstra S, Ghodousi J, Ahmadi H (2010). SWAT-simulated hydrological impact of landuse change in the Zanjanrood Basin, Northwest Iran. Hydrological Processes 24(7):892-903

Guevara-Escobar A, González-Sosa E, Véliz-Chávez C, Ventura-Ramos E, Ramos-Salinas M (2007). Rainfall interception and distribution patterns of gross precipitation around an isolated Ficus benjamina tree in an urban area. Journal of Hydrology 333(2):532-541

Han S, Yang Y, Fan T, Xiao D, Moiwo JP, 2012. Precipitation-runoff processes in Shimen hillslope micro-catchment of Taihang Mountain, north China. Hydrological Processes 26(9):1332-1341

Harden CP (1991). Land-use, soil-erosion, and reservoir sedimentation in an Andean drainage basin in Ecuador. International Workshop and Field Excursions: Mountain Geoecology of the Andes Santiago, Chile, pp. 177-184

Haverkamp S, Fohrer N, Frede H-G (2005). Assessment of the effect of land use patterns on hydrologic landscape functions: a comprehensive GIS-based tool to minimize model uncertainty resulting from spatial aggregation. Hydrological Processes 19(3):715-727

Hofstede R (1998). Impactos ecológicos de plantaciones forestales. In: II Conferencia Electrónica sobre Usos Sostenibles y Conservación del Ecosistema Páramo en los Andes, Disponible en http://infoandina.mtnforum.org/sites/default/files/publication /files/Impactos_ecol_gicos_de_plantaciones_forestales.pdf, 9 pp

Hofstede R (2011). Un árbol no siempre es más agua: a propósito de las políticas de (re) forestación. CONDESAN, Propuestas Andinas, \#3, 3 pp

Jiménez F (2005). El bosque como regulador del ciclo hidrológico. Centro Agronómico Tropical de Investigación Y Enseñanza, CATIE. Costa Rica. Disponible en http://www.inecc.gob.mx/descargas/dgipea/fran_jimenez.pdf, 25 pp

Knox JC (2001). Agricultural influence on landscape sensitivity in the Upper Mississippi River Valley. Catena 42(2):193-224

Kotei R, Kyei-Baffour N, Ofori E, Agyare W (2013). Changes in the Sumampa streamflow flashiness in the forest-savannah transitional zone, Mampong-Ashant, Ghana 1985-2009. Journal of Engineering and Applied Sciences 8(9):770-778

Krajenbrink HJ (2007). Application of SWAT for rainfall-runoff modelling in small tropical mountainous catchments in Ecuador. MSc Thesis, Wageningen University, The Netherlands 
Lahmer W, Pfutzner B, Becker A, 2001. Assessment of land use and climate change impacts on the mesoscale. Physics and Chemistry of the Earth, Part B: Hydrology, Oceans and Atmosphere 26(7-8):565-575

Li T, Gao Y (2015). Runoff and sediment yield variations in response to precipitation changes: A case study of Xichuan watershed in the loess plateau, China. Water 7(10):5638-5656

Li Z, Liu W, Zhang X, Zheng F (2009). Impacts of land use change and climate variability on hydrology in an agricultural catchment on the Loess Plateau of China. Journal of Hydrology 377(1):35-42

Lin Y-P, Hong N-M, Wu P-J, Wu C-F, Verburg PH (2007). Impacts of land use change scenarios on hydrology and land use patterns in the $\mathrm{Wu}-\mathrm{Tu}$ watershed in Northern Taiwan. Landscape and Urban Planning 80(1):111-126

Lørup JK, Refsgaard JC, Mazvimavib D (1998). Assessing the effect of land use change on catchment runoff by combined use of statistical tests and hydrological modelling: Case studies from Zimbabwe. Journal of Hydrology 205:147-163

Mao D, Cherkauer KA (2009). Impacts of land-use change on hydrologic responses in the Great Lakes region. Journal of Hydrology 374(1):71-82

Martínez-Zavala L, Jordán A (2008). Effect of rock fragment cover on interrill soil erosion from bare soils in Western Andalusia, Spain. Soil Use and Management 24(1):108-117

Molina A, Govers G, Vanacker V, Poesen J, Zeelmaekers E, Cisneros F (2007). Runoff generation in a degraded Andean ecosystem: Interaction of vegetation cover and land use. Catena 71:357-370

Molina A, Vanacker V, Balthazar V, Mora D, Govers G (2012). Complex land cover change, water and sediment yield in a degraded Andean environment. Journal of Hydrology 472-473:25-35

Nanzyo M, Shoji S, Dahlgren R (1993). Physical characteristics of volcanic ash soils. In: Shoji, S., M. Nanzyo, R. Dahlgren (Eds.), Volcanic ash soils: genesis, properties and utilization. Amsterdam, The Netherlands: Elsevier Science Publishers B.V., 288 pp

Neris J, Tejedor M, Rodríguez M, Fuentes J, Jiménez C (2013). Effect of forest floor characteristics on water repellency, infiltration, runoff and soil loss in Andisols of Tenerife (Canary Islands, Spain). Catena 108:50-57

OEHHA (2015). Flashiness indicator. Recuperado de http://oehha.ca.gov/ecotox/drycreek/ Flashiness_2015.pdf

Pan Z, Takle E, Segal M, Anritt R (1999). Simulation of potential impacts of manmade land use changes on US summer climate under various synoptic regimes. Journal of Geophysical Research 104:6515-6528

Perrin JL, Bouvier C, Janeau JL, Menez G, Cruz F (2001). Rainfall/runoff processes in a small periurban catchment in the Andes mountains. The Rumihurcu Quebrada, Quito (Ecuador). Hydrological Processes 15:843-854

Prowse TD, Beltaos D, Gardner JT, Gibson JJ, Granger RJ, Leconte R, Peters DL, Pietroniro A, Romolo LA, Toth B (2006). Climate change, flow regulation and land-use effects on the hydrology of the Peace-Athabasca-Slave system; findings from the northern rivers ecosystem initiative. Environmental Monitoring and Assessment 113(1-3):167-197

Rutter AJ (1959). Evaporation from a plantation of pinus sylvestris in relation to meteorological and soil conditions. Available at http://hydrologie.org/redbooks/a048/048012.pdf, pp. 101-110

Rutter AJ (1963). Studies in the water relations of Pinus Sylvestris in plantation conditions I. Measurements of rainfall and interception. Journal of Ecology 51(1):191-203

Rutter AJ (1967). An analysis of evaporation from a stand of Scots pine. Forest Hydrology 403:417

Scott DF, Bruijnzeel LA, Mackensen J, 2005. The hydrological and soil impacts of forestation in the tropics. In: Bonell, M., L.A. Bruijnzeel (Eds.), Forests, Water and People in the Humid Tropics, Chap. 25, pp. 622-651

Sriwongsitanon N, Taesombat W (2011). Effects of land cover on runoff coefficient. Journal of Hydrology 410(3):226-238 
Tollan A (2002). Land-use change and floods: what do we need most, research or management? Water Science and Technology 45(8):183-190

Tomer MD, Schilling KE (2009). A simple approach to distinguish land-use and climate-change effects on watershed hydrology. Journal of Hydrology 376(1):24-33

Twine TE, Kucharik CJ, Foley JA (2004). Effects of Land Cover Change on the Energy and Water Balance of the Mississippi River Basin. Journal of Hydrometeorology 5(4):640-655

Vuille M (2013). El cambio climático y los recursos hídricos en los Andes tropicales. BID, Disponible en http://facets.sdsu.edu/Glaciar_CCRRR.pdf, 33 pp

Wagner PD, Kumar S, Schneider K (2013). An assessment of land use change impacts on the water resources of the Mula and Mutha Rivers catchment upstream of Pune, India. Hydrology and Earth System Sciences 17(6):2233-2246

Wang GX, Zhang Y, Liu GM, Chen L (2006). Impact of land use change on hydrological processes in the Maying River basin, China. Science in China Serie D-Earth Sciences 49(10):1098-1110

Willems P (2004). WETSPRO: Water Engineering Time Series PROcessing tool. KU Leuven Hydraulics Laboratory, Leuven Belgium. Manual available at http://www.kuleuven.be/hydr/pw/WETSPRO\%20manual.pdf, 22 pp

Woods SW, Balfour VN (2008). The effect of ash on runoff and erosion after a severe forest wildfire, Montana, USA. International Journal of Wildland Fire 17:535-548

Woods SW, Balfour VN (2010). The effects of soil texture and ash thickness on the post-fire hydrological response from ash covered soils. Journal of Hydrology 393:274-286

Zavala LM, Jordán A, Gil J, Bellifante N, Pain C (2009). Intact ash and charred litter reduces susceptibility to rain splash erosion post-wildfire. Earth Surface Processes and Landforms 34:1522-1532 